\title{
Further Results on Pair Sum Graphs
}

\author{
Raja Ponraj $^{1}$, Jeyaraj Vijaya Xavier Parthipan ${ }^{2}$, Rukhmoni Kala ${ }^{3}$ \\ ${ }^{1}$ Department of Mathematics, Sri Paramakalyani College, Alwarkurichi, India \\ ${ }^{2}$ Department of Mathematics, St. John's College, Palayamcottai, India \\ ${ }^{3}$ Department of Mathematics, Manonmaniam Sundaranar University, Tirunelveli, India \\ Email: ponrajmaths@indiatimes.com, parthi68@rediffmail.com,karthipyi91@yahoo.co.in
}

Received January 3, 2012; revised February 9, 2012; accepted February 16, 2012

\begin{abstract}
Let $G$ be a $(p, q)$ graph. An injective map $f: V(G) \rightarrow\{ \pm 1, \pm 2, \cdots, \pm p\}$ is called a pair sum labeling if the induced edge function, $f_{e}: E(G) \rightarrow Z-\{0\}$ defined by $f_{e}(u v)=f(u)+f(v)$ is one-one and $f_{e}(E(G))$ is either of the form $\left\{ \pm k_{1}, \pm k_{2}, \cdots, \pm k_{q / 2}\right\}$ or $\left\{ \pm k_{1}, \pm k_{2}, \cdots, \pm k_{(q-1) / 2}\right\} \cup\left\{k_{(q+1) / 2}\right\}$ according as $q$ is even or odd. A graph with a pair sum labeling is called a pair sum graph. In this paper we investigate the pair sum labeling behavior of subdivision of some standard graphs.
\end{abstract}

Keywords: Path; Cycle; Ladder; Triangular Snake; Quadrilateral Snake

\section{Introduction}

The graphs considered here will be finite, undirected and simple. $V(G)$ and $E(G)$ will denote the vertex set and edge set of a graph $G$. The cardinality of the vertex set of a graph $G$ is denoted by $p$ and the cardinality of its edge set is denoted by $q$. The corona $G_{1} G_{2}$ of two graphs $G_{1}$ and $G_{2}$ is defined as the graph obtained by taking one copy of $G_{1}$ (with $p_{1}$ vertices) and $p_{1}$ copies of $G_{2}$ and then joining the ith vertex of $G_{1}$ to all the vertices in the ith copy of $G_{2}$. If $e=u v$ is an edge of $G$ and $w$ is a vertex not in $G$ then $e$ is said to be subdivided when it is replaced by the edges $u w$ and $w v$. The graph obtained by subdividing each edge of a graph $G$ is called the subdivision graph of $G$ and it is denoted by $S(G)$. The graph $P_{n} \times P_{2}$ is called the ladder. A dragon is a graph formed by joining an end vertex of a path $P_{m}$ to a vertex of the cycle $C_{n}$. It is denoted as $C_{n} @ P_{m}$. The triangular snake $T_{n}$ is obtained from the path $P_{n}$ by replacing every edge of a path by a triangle $C_{3}$. The quadrilateral snake $Q_{n}$ is obtained from the path $P_{n}$ by every edge of a path is replaced by a cycle $C_{4}$. The concept of pair sum labeling has been introduced in [1]. The Pair sum labeling behavior of some standard graphs like complete graph, cycle, path, bistar, and some more standard graphs are investigated in [1-3]. That all the trees of order $\leq 9$ are pair sum have been proved in [4]. Terms not defined here are used in the sense of Harary [5]. Let $x$ be any real number. Then $\lfloor x\rfloor$ stands for the largest integer less than or equal to $x$ and $\lceil x\rceil$ stands for the smallest integer greater than or equal to $x$. Here we investigate the pair sum labeling behavior of $S(G)$, for some standard graphs $G$.

\section{Pair Sum Labeling}

Definition 2.1. Let $G$ be a $(p, q)$ graph. An injective map $f: V(G) \rightarrow\{ \pm 1, \pm 2, \cdots, \pm p\}$ is called a pair sum labeling if the induced edge function, $f_{e}: E(G) \rightarrow Z-\{0\}$ defined by $f_{e}(u v)=f(u)+f(v)$ is one-one and $f_{e}(E(G))$ is either of the form

$$
\left\{ \pm k_{1}, \pm k_{2}, \cdots, \pm k_{q / 2}\right\}
$$

or

$$
\left\{ \pm k_{1}, \pm k_{2}, \cdots, \pm k_{(q-1) / 2}\right\} \cup\left\{k_{(q+1) / 2}\right\}
$$

according as $q$ is even or odd. A graph with a pair sum labeling defined on it is called a pair sum graph.

Theorem 2.2 [1]. Any path is a pair sum graph.

Theorem 2.3 [1]. Any cycle is a pair sum graph.

\section{On Standard Graphs}

Here we investigate pair sum labeling behavior of $C_{n} @ P_{m}$ and $K_{n}^{c}+2 K_{2}$.

Theorem 3.1. If $n$ is even, $C_{n} @ P_{m}$ is a pair sum graph.

Proof. Let $C_{n}$ be the cycle $u_{1} u_{2} u_{3} \cdots u_{n} u_{1}$ and let $P_{m}$ be the path $v_{1} v_{2} \cdots v_{m}$.

Case 1. $m \equiv 0(\bmod 4)$ 
Define

$$
f: V\left(C_{n} @ P_{m}\right) \rightarrow\{ \pm 1, \pm 2, \cdots, \pm(m+n)\}
$$

by

$$
\begin{aligned}
& f\left(v_{\lfloor m / 2\rfloor-i+1}\right)=i, 1 \leq i \leq\lfloor m / 2\rfloor \\
& f\left(v_{\lfloor m / 2\rfloor+2 i-1}\right)=-2 i-2,1 \leq i \leq\lfloor m / 4\rfloor \\
& f\left(v_{\lfloor m / 2\rfloor+2 i}\right)=-2 i+1,1 \leq i \leq\lfloor m / 4\rfloor \\
& f\left(u_{i}\right)=m / 2+2 i-1,1 \leq i \leq n / 2 \\
& f\left(u_{n / 2+i}\right)=-m / 2-2 i+1,1 \leq i \leq n / 2 .
\end{aligned}
$$

Here

$$
\begin{aligned}
& f_{e}\left(E\left(C_{n} @ P_{m}\right)\right) \\
& =\{3,5,7, \cdots,(m+1)\} \cup\{-3,-5, \cdots,-(m+1)\} \\
& \cup\{m+4, m+8, \cdots,(m+2 n-4)\} \\
& \cup\{-(m+4),-(m+8), \cdots,-(m+2 n-4)\} \\
& \cup\{n-2,-(n-2)\} .
\end{aligned}
$$

Therefore $f$ is a pair sum labeling.

Case 2. $m \equiv 2(\bmod 4)$

Define

$$
f: V\left(C_{n} @ P_{m}\right) \rightarrow\{ \pm 1, \pm 2, \cdots, \pm(m+n)\}
$$

by

$$
\begin{aligned}
& f\left(v_{\lfloor m / 2\rfloor-2 i}\right)=1-2 i, 1 \leq i \leq\lfloor(m+2) / 4\rfloor \\
& f\left(v_{\lfloor m / 2\rfloor-2 i+1}\right)=-2 i-2,1 \leq i \leq\lfloor(m-2) / 4\rfloor \\
& f\left(v_{\lfloor m / 2\rfloor+i-1}\right)=i, 1 \leq i \leq\lceil m / 2\rceil+1 \\
& f\left(u_{i}\right)=-\lfloor m / 2\rfloor-2 i-1,1 \leq i \leq n / 2 \\
& f\left(u_{n / 2+i}\right)=\lfloor m / 2\rfloor+2 i+1,1 \leq i \leq n / 2
\end{aligned}
$$

Here

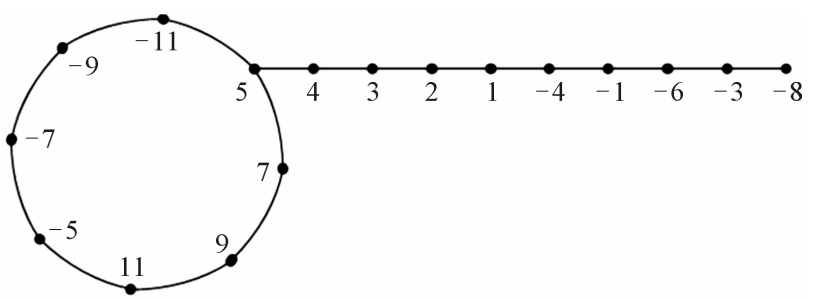

Figure 1. A pair sum labeling of $C_{8} @ P_{9}$.

$$
\begin{aligned}
& f_{e}\left(E\left(C_{n} @ P_{m}\right)\right) \\
& =\{-3,-5,-7, \cdots,-m,-(m+1)\} \\
& \cup\{3,5,7, \cdots,(m+1)\} \\
& \cup\{m+2, m+10, \cdots,(m+2 n)\} \\
& \cup\{-(m+2),-(m+10), \cdots,-(m+2 n)\} \\
& \cup\{(n-2),-(n-2)\} .
\end{aligned}
$$

Hence $f$ is a pair sum labeling.

Case 3. $m \equiv 1(\bmod 4)$

Label the vertex $u_{i}(1 \leq i \leq n), v_{i}(1 \leq i \leq m-1)$ as in Case 1. Then label $-m-2$ to $v_{m}$.

Case 4. $m \equiv 3(\bmod 4)$

Assign the label $m+2$ to $v_{m}$ and assign the label to the remaining vertices as in Case 2 .

Illustration 1. A pair sum labeling of $C_{8} @ P_{9}$ is shown in Figure 1.

Theorem 3.2. $K_{n}^{c}+2 K_{2}$ is pair sum graph if $n$ is even.

Proof: Let $u_{1}, u_{2}, \cdots, u_{n}$ be the vertices of $K_{n}$ and $u$, $v, w, z$. be the vertices in $2 K_{2}$. Let

$$
V\left(K_{n}^{c}+2 K_{2}\right)=V\left(K_{n}^{c}\right) \cup V\left(2 K_{2}\right)
$$

and

$$
E\left(K_{n}^{c}+2 K_{2}\right)=\left\{u v, w z, u u_{i}, v u_{i}, w u_{i}, z u_{i}: 1 \leq i \leq n\right\} .
$$

Define

$$
f: V\left(K_{n}^{c}+2 K_{2}\right) \rightarrow\{ \pm 1, \pm 2, \cdots, \pm(n+4)\}
$$

by

$$
\begin{aligned}
& f\left(u_{i}\right)=2 i-1,1 \leq i \leq n / 2 \\
& f\left(u_{n / 2+i}\right)=-(2 i-1), 1 \leq i \leq n / 2 \\
& f(u)=n, f(v)=n+3 \\
& f(w)=-n, f(z)=-(n+3)
\end{aligned}
$$

Here

$$
\begin{aligned}
& f_{e}\left(E\left(K_{n}^{c}+2 K_{2}\right)\right) \\
& =\{n+1, n+3, n+5, \cdots, 2 n\} \\
& \cup\{-(n+1),-(n+3),-(n+5), \cdots,-2 n\} \\
& \cup\{n-1, n-3, n-5, \cdots, 1\} \\
& \cup\{-(n-1),-(n-3),-(n-5), \cdots,-1\} \\
& \cup\{n+4, n+8, n+12, \cdots, 2 n+2\} \\
& \cup\{-(n+4),-(n+8),-(n+12), \cdots,-(2 n+2)\} \\
& \cup\{n+2, n, n-2, \cdots, 2\} \\
& \cup\{-(n+2),-n,-(n-2), \cdots,-2\} \\
& \cup\{2 n+3,-(2 n+3)\} .
\end{aligned}
$$


Therefore $f$ is a pair sum labeling.

Illustration 2. A pair sum labeling of $K_{8}^{c}+2 K_{2}$ is shown in Figure 2.

\section{On Subdivision Graph}

Here we investigate the pair sum labeling behavior of $S(G)$ for some standard graphs $G$.

Theorem 4.1. $S\left(L_{n}\right)$ is a pair sum graph, where $L_{n}$ is a ladder on $n$ vertices.

Proof. Let

$$
V\left(S\left(L_{n}\right)\right)=\left\{u_{i}, v_{i}, w_{i}, a_{j}, b_{j}: 1 \leq i \leq n, 1 \leq j \leq n-1\right\}
$$

Let

$$
\begin{aligned}
E\left(S\left(L_{n}\right)\right) & =\left\{u_{i} w_{i}, w_{i} v_{i}: 1 \leq i \leq n\right\} \\
& \cup\left\{u_{i} a_{i} a_{i} u_{i+1}, v_{i} b_{i}, b_{i} v_{i+1}: 1 \leq i \leq n-1\right\} .
\end{aligned}
$$

Case 1: $n$ is even.

When $n=2$, the proof follows from the Theorem 2.3.

For $n>2$,

Define

$$
f: V\left(S\left(L_{n}\right)\right) \rightarrow\{ \pm 1, \pm 2, \pm 3, \cdots, \pm(5 n-2)\}
$$

by

$$
\begin{aligned}
& f\left(u_{n / 2}\right)=-1, f\left(u_{n / 2+1}\right)=-3 \\
& f\left(u_{n / 2-i}\right)=10 i+3,1 \leq i \leq(n-2) / 2 \\
& f\left(u_{n / 2+i+1}\right)=-10 i+1,1 \leq i \leq(n-2) / 2 \\
& f\left(w_{n / 2}\right)=5, f\left(w_{n / 2+1}\right)=-5 \\
& f\left(w_{n / 2-i}\right)=10 i+1,1 \leq i \leq(n-2) / 2 \\
& f\left(w_{n / 2+1+i}\right)=-(10 i+1), 1 \leq i \leq(n-2) / 2 \\
& f\left(v_{n / 2}\right)=3, f\left(v_{n / 2+1}\right)=1 \\
& f\left(v_{n / 2-i}\right)=10 i-1,1 \leq i \leq(n-2) / 2 \\
& f\left(v_{n / 2+1+i}\right)=-10 i-3,1 \leq i \leq(n-2) / 2 \\
& f\left(a_{n / 2}\right)=-2 \\
& f\left(a_{n / 2-i}\right)=10 i+5,1 \leq i \leq(n-2) / 2 \\
& f\left(a_{n / 2+i}\right)=-10 i+3,1 \leq i \leq(n-2) / 2 \\
& f\left(b_{n / 2}\right)=2 \\
& f\left(b_{n / 2-i}\right)=10 i-3,1 \leq i \leq(n-2) / 2 \\
& f\left(b_{n / 2+i+1}\right)=-10 i-5,1 \leq i \leq(n-2) / 2 .
\end{aligned}
$$

When $n=4$,

$$
\begin{aligned}
& f_{e}\left(E\left(S\left(L_{n}\right)\right)\right)=\{3,4,5,8,10,16,20,24,28\} \\
& \cup\{-3,-4,-5,-8,-10,-16,-20,-24,-28\} .
\end{aligned}
$$

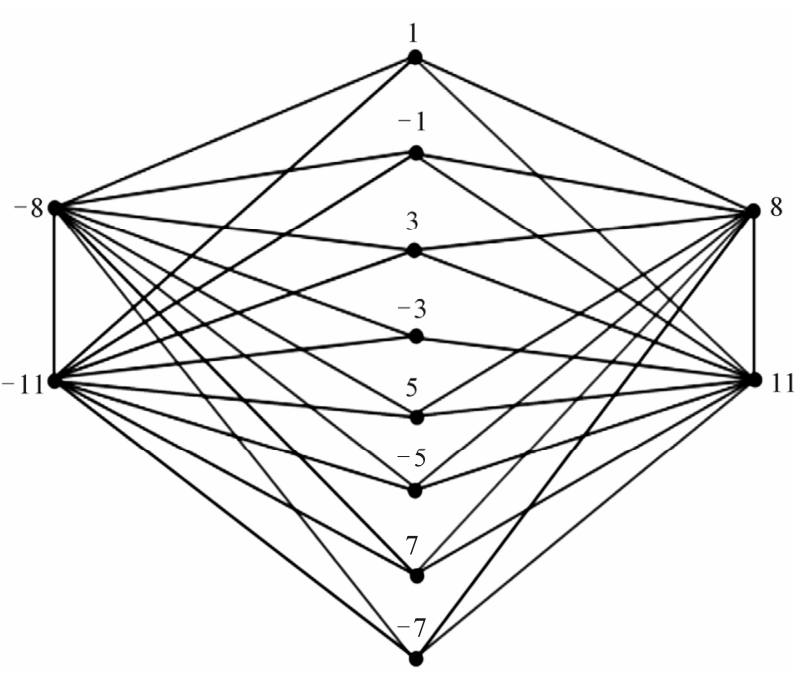

Figure 2. A pair sum labeling of $K_{8}^{c}+2 K_{2}$.

For $n>4$,

$$
\begin{aligned}
& f_{e}\left(E\left(S\left(L_{n}\right)\right)\right) \\
&=f_{e}\left(E\left(S\left(L_{4}\right)\right)\right) \cup\{(26,36,40,44,48,38), \\
&(-26,-36,-40,-44,-48,-38), \\
&(46,56,60,64,68,58), \\
&(-46,-56,-60,-64,-68,-58), \cdots, \\
&(10 n-34,10 n-24,10 n-20, \\
&10 n-16,10 n-12,10 n-22), \\
&(-10 n+34,-10 n+24,-10 n+20, \\
&-10 n+16,-10 n+12,-10 n+22)\} .
\end{aligned}
$$

Therefore $f$ is a pair sum labeling.

Case 2. $n$ is odd.

Clearly $S\left(L_{1}\right) \cong P_{3}$ and hence $S\left(L_{n}\right)$ is a pair sum graph by Theorem 2.2. For $n>1$,

Define

$$
f: V\left(S\left(L_{n}\right)\right) \rightarrow\{ \pm 1, \pm 2, \cdots, \pm(5 n-2)\}
$$

by

$$
\begin{aligned}
& f\left(u_{(n+1) / 2}\right)=6, f\left(u_{(n-1) / 2}\right)=12 \\
& f\left(u_{(n+3) / 2}\right)=-12, f\left(a_{(n-1) / 2}\right)=-9 \\
& f\left(a_{(n+1) / 2}\right)=3 \\
& f\left(u_{(n+3) / 2+i}\right)=10 i+10,1 \leq i \leq(n-3) / 2 \\
& f\left(u_{(n-1) / 2-i}\right)=-(10 i+10), 1 \leq i \leq(n-3) / 2 \\
& f\left(v_{(n+3) / 2+i}\right)=-(6+10 i), 1 \leq i \leq(n-3) / 2
\end{aligned}
$$




$$
\begin{aligned}
& f\left(v_{(n-1) / 2-i}\right)=6+10 i, 1 \leq i \leq(n-3) / 2 \\
& f\left(w_{(n+3) / 2+i}\right)=-10 i+2,1 \leq i \leq(n-3) / 2 \\
& f\left(w_{(n-1) / 2-i}\right)=10 i-2,1 \leq i \leq(n-3) / 2 \\
& f\left(v_{(n+1) / 2}\right)=2, f\left(v_{(n-1) / 2}\right)=10 \\
& f\left(v_{(n+3) / 2}\right)=-10, f\left(b_{(n-1) / 2}\right)=-6 \\
& f\left(b_{(n+1) / 2}\right)=4, f\left(w_{(n+1) / 2}\right)=-4 \\
& f\left(w_{(n-1) / 2}\right)=8, f\left(w_{(n+1) / 2}\right)=-8 \\
& f\left(a_{(n+1) / 2+i}\right)=-(10 i+12), 1 \leq i \leq(n-3) / 2 \\
& f\left(a_{(n-1) / 2-i}\right)=10 i+12,1 \leq i \leq(n-3) / 2 \\
& f\left(b_{(n+1) / 2+i}\right)=-(10 i+4), 1 \leq i \leq(n-3) / 2 \\
& f\left(b_{(n-1) / 2-i}\right)=10 i+4,1 \leq i \leq(n-3) / 2 .
\end{aligned}
$$

Therefore

$$
\begin{aligned}
& f_{e}\left(E\left(S\left(L_{3}\right)\right)\right) \\
& =\{2,3,4,6,9,18,20,-2,-3,-4,-6,-9,-18,-20\}
\end{aligned}
$$

and

$$
\begin{aligned}
& f_{e}\left(E\left(S\left(L_{5}\right)\right)\right)=f_{e}\left(E\left(S\left(L_{3}\right)\right)\right) \\
& \cup\{24,30,34,38,42,36,-24,-30,-34,-38,-42,-36\}
\end{aligned}
$$

when $n>5$,

$$
\begin{aligned}
& f_{e}\left(E\left(S\left(L_{n}\right)\right)\right) \\
&=f_{e}\left(E\left(S\left(L_{5}\right)\right)\right) \cup\{(40,50,54,58,62,52), \\
&(-40,-50,-54,-58,-62,-52), \\
&(60,70,74,78,82,72), \\
&(-60,-70,-74,-78,-82,-72), \ldots, \\
&(10 n-30,10 n-20,10 n-16, \\
&10 n-12,10 n-8,10 n-18), \\
&(-10 n+30,-10 n+20,-10 n+16, \\
&-10 n+12,-10 n+8,-10 n+18)\} .
\end{aligned}
$$

Then $f$ is a pair sum labeling.

Illustration 3. A pair sum labeling of $S\left(L_{7}\right)$ is shown in Figure 3.

Theorem 4.2. $S\left(C_{n} K_{1}\right)$ is a pair sum graph

Proof. Let

$$
V\left(S\left(C_{n} K_{1}\right)\right)=\left\{u_{i}: 1 \leq i \leq 2 n\right\} \cup\left\{w_{i}, v_{i}: 1 \leq i \leq n\right\}
$$

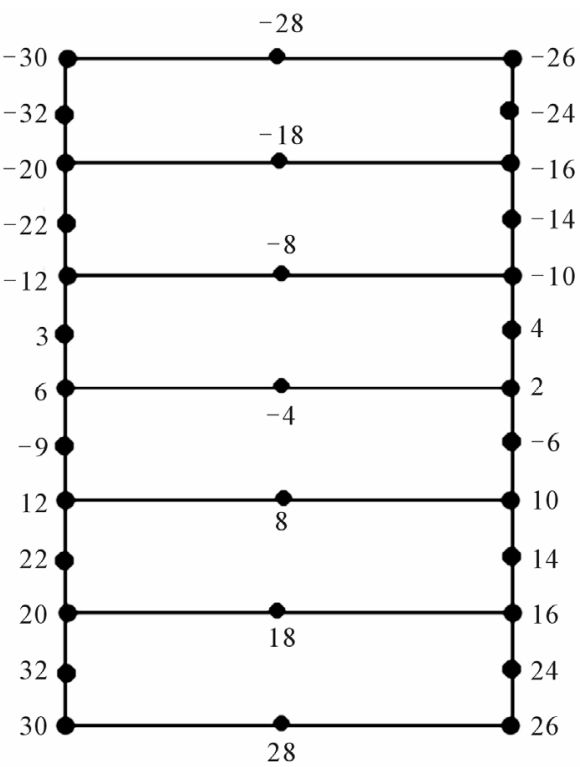

Figure 3. A pair sum labeling of $S\left(L_{7}\right)$.

Let

$$
\begin{aligned}
& E\left(S\left(C_{n} K_{1}\right)\right)=\left\{u_{i} u_{i+1}: 1 \leq i \leq 2 n-1\right\} \\
& \cup\left\{u_{2 i-1} w_{i}: 1 \leq i \leq n\right\} \cup\left\{v_{i} w_{i}: 1 \leq i \leq n\right\} .
\end{aligned}
$$

Case 1. $n$ is even.

Define

$$
f: S\left(C_{n} K_{1}\right) \rightarrow\{ \pm 1, \pm 2, \cdots, \pm 4 n\}
$$

by

$$
\begin{aligned}
& f\left(u_{i}\right)=2 i-1,1 \leq i \leq n \\
& f\left(u_{n+i}\right)=-(2 i-1), 1 \leq i \leq n \\
& f\left(w_{i}\right)=2 n-1+2 i, 1 \leq i \leq n / 2 \\
& f\left(w_{n / 2+i}\right)=-2 n+1-2 i, 1 \leq i \leq n / 2 \\
& f\left(v_{i}\right)=3 n-1-2 i, 1 \leq i \leq n / 2 \\
& f\left(w_{n / 2+i}\right)=-3 n+1+2 i, 1 \leq i \leq n / 2
\end{aligned}
$$

Here

$$
\begin{aligned}
& f_{e}(E)=\{4,8,12, \cdots,(4 n-4)\} \\
& \cup\{-4,-8,-12, \cdots,-(4 n-4)\} \\
& \cup\{2 n+2,2 n+8,2 n+14, \cdots, 5 n-4\} \\
& \cup\{-(2 n+2),-(2 n+8),-(2 n+14), \cdots,-(5 n-4)\} \\
& \cup\{5 n+2,5 n+6,5 n+10, \cdots, 7 n-2\} \\
& \cup\{-(5 n+2),-(5 n+6),-(5 n+10), \cdots,-(7 n-2)\} .
\end{aligned}
$$

Then $f$ is pair sum labeling.

Case 2. $n$ is odd.

Define 


$$
f: V\left(S\left(C_{n} K_{1}\right)\right) \rightarrow\{ \pm 1, \pm 2, \cdots, \pm 4 n\}
$$

by

$$
\begin{aligned}
& f\left(u_{i}\right)=4 n-2 i+2,1 \leq i \leq n \\
& f\left(u_{n / 2+i}\right)=-4 n+2 i-2,1 \leq i \leq n \\
& f\left(w_{i}\right)=-n-1+i, 1 \leq i \leq\lceil n / 2\rceil \\
& f\left(w_{\lceil n / 2\rceil+i}\right)=n-i, 1 \leq i \leq\lfloor n / 2\rfloor \\
& f\left(v_{i}\right)=-2 n-2+2 i, 1 \leq i \leq\lceil n / 2\rceil \\
& f\left(v_{\lceil n / 2\rceil+i}\right)=2 n+2-2 i, 1 \leq i \leq\lfloor n / 2\rfloor
\end{aligned}
$$

Here

$$
\begin{aligned}
& f_{e}\left(E\left(S\left(C_{n} K_{1}\right)\right)\right) \\
& =\{8 n-2,8 n-6, \cdots 4 n+10,40+6\} \\
& \cup\{-(8 n-2),-(8 n-6), \cdots,-(4 n+10),-(4 n+6)\} \\
& \cup\{2 n-2,-2 n+2\} \\
& \cup\{3 n, 3 n-3,3 n-6, \cdots, 3(n+1) / 2\} \\
& \cup\{-3 n,-(3 n-3),-(3 n-6), \cdots,-3(n+1) / 2\} \\
& \cup\{3 n-1,3 n-4, \cdots, 3(n+7) / 2\} \\
& \cup\{-(3 n-1),-(3 n-4), \cdots,-3(n+7) / 2\} .
\end{aligned}
$$

Then $f$ is pair sum labeling.

Illustration 4. A pair sum labeling of $S\left(C_{7} K_{1}\right)$ is shown in Figure 4.

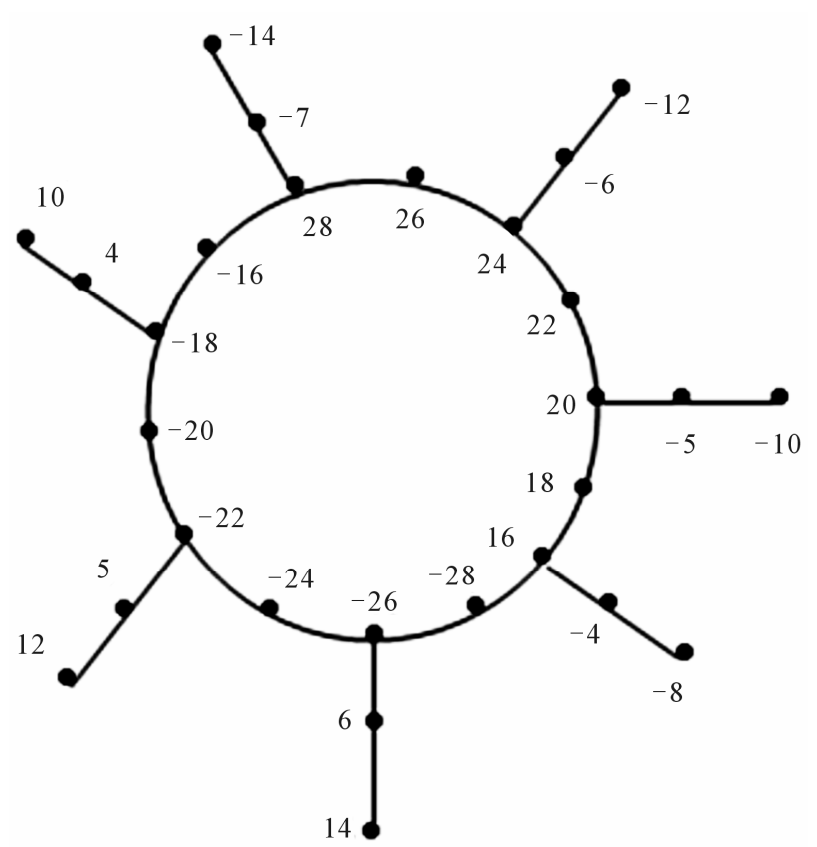

Figure 4. A pair sum labeling of $S\left(C_{7} K_{1}\right)$.
Theorem 4.3. $S\left(P_{n} K_{1}\right)$ is a pair sum graph. Proof. Let

$$
V\left(S\left(P_{n} K_{1}\right)\right)=\left\{u_{i}: 1 \leq i \leq 2 n-1\right\} \cup\left\{w_{i}, v_{i}: 1 \leq i \leq n\right\}
$$

Let

$$
\begin{aligned}
& E\left(S\left(P_{n} K_{1}\right)\right)=\left\{u_{i} u_{i+1}: 1 \leq i \leq 2 n-2\right\} \\
& \cup\left\{u_{2 i-1} w_{i}: 1 \leq i \leq n\right\} \cup\left\{v_{i} w_{i}: 1 \leq i \leq n\right\} .
\end{aligned}
$$

Case 1. $n$ is even.

When $n=2$, the proof follows from Theorem 2.2. For $n>2$, Define

$$
f: V\left(S\left(P_{n} K_{1}\right)\right) \rightarrow\{ \pm 1, \pm 2, \cdots, \pm(4 n-1)\}
$$

by

$$
\begin{aligned}
& f\left(u_{n}\right)=1, f\left(u_{n-1}\right)=-2 \\
& f\left(u_{n+1}\right)=2 \\
& f\left(u_{n-1-2 i}\right)=-5 i-2,1 \leq i \leq n / 2-1 \\
& f\left(u_{n-2 i}\right)=-(5 i+3), 1 \leq i \leq n / 2-1 \\
& f\left(u_{n+1+2 i}\right)=5 i+3,1 \leq i \leq n / 2-1 \\
& f\left(u_{n+2 i}\right)=5 i+2,1 \leq i \leq n / 2-1 \\
& f\left(w_{n / 2}\right)=4, f\left(w_{n / 2+1}\right)=-5 \\
& f\left(w_{n / 2-i}\right)=-5 i-4,1 \leq i \leq(n-2) / 2 \\
& f\left(w_{n / 2+i+1}\right)=5 i+4,1 \leq i \leq(n-2) / 2 \\
& f\left(v_{n / 2}\right)=-6, f\left(v_{n / 2+1}\right)=6 \\
& f\left(v_{n / 2-i}\right)=-5 i-5,1 \leq i \leq(n-2) / 2 \\
& f\left(v_{n / 2+i+1}\right)=5 i+5,1 \leq i \leq(n-2) / 2
\end{aligned}
$$

Here

$$
\begin{aligned}
& f_{e}\left(E\left(S\left(P_{4} K_{1}\right)\right)\right)=\{1,2,3,9,15,17,19\} \\
& \cup\{-1,-2,-3,-9,-15,-17,-19\} .
\end{aligned}
$$

For $n>4$,

$$
\begin{aligned}
& f_{e}\left(E\left(S\left(P_{n} K_{1}\right)\right)\right)=f_{e}\left(E\left(S\left(P_{4} K_{1}\right)\right)\right) \\
& \cup\{20,25,27,29\} \cup\{-20,-25,-27,-29\} \\
& \cup\{30,35,37,39\} \cup\{-30,-35,-37,-39\} \cup, \cdots, \\
& \cup\{5 n-10,5 n-5,5 n-3,5 n-1\} \\
& \cup\{-(5 n-10),-(5 n-5),-(5 n-3),-(5 n-1)\} .
\end{aligned}
$$

Then $f$ is pair sum labeling.

Case 2. $n$ is odd.

Since $S\left(P_{1} K_{1}\right) \cong P_{3}$, which is a pair sum graph by Theorem 2.3. For $n>1$, Define 


$$
f: V\left(S\left(P_{n} K_{1}\right)\right) \rightarrow\{ \pm 1, \pm 2, \cdots, \pm(4 n-1)\}
$$

by

$$
\begin{aligned}
& f\left(u_{(n+1) / 2}\right)=1, f\left(u_{(n-1) / 2}\right)=8 \\
& f\left(u_{(n+3) / 2}\right)=-8 \\
& f\left(u_{(n-1) / 2-2 i}\right)=-10 i+1,1 \leq i \leq\lfloor n / 2\rfloor-1 \\
& f\left(u_{(n+1) / 2-2 i}\right)=5 i+5,1 \leq i \leq\lfloor n / 2\rfloor-1 \\
& f\left(u_{(n+3) / 2+2 i}\right)=-10 i-1,1 \leq i \leq\lfloor n / 2\rfloor-1 \\
& f\left(u_{(n+1) / 2+2 i}\right)=-(5 i+5), 1 \leq i \leq\lfloor n / 2\rfloor-1 \\
& f\left(w_{(n+1) / 2}\right)=-2, f\left(w_{(n-1) / 2}\right)=-5 \\
& f\left(w_{(n+3) / 2}\right)=6 \\
& f\left(w_{(n-1) / 2-i}\right)=5 i+7,1 \leq i \leq\lfloor n / 2\rfloor-1 \\
& f\left(w_{(n+3) / 2+i}\right)=-(5 i+7), 1 \leq i \leq\lfloor n / 2\rfloor-1 \\
& f\left(v_{(n+1) / 2}\right)=3, f\left(v_{(n-1) / 2}\right)=9 \\
& f\left(v_{(n+3) / 2}\right)=-9 \\
& f\left(v_{(n-1) / 2-i}\right)=5 i+8,1 \leq i \leq\lfloor n / 2\rfloor-1 \\
& f\left(v_{(n+3) / 2+i}\right)=-(5 i+8), 1 \leq i \leq\lfloor n / 2\rfloor-1 \\
&
\end{aligned}
$$

Here

$$
\begin{aligned}
& f_{e}\left(E\left(S\left(P_{3} K_{1}\right)\right)\right)=\{1,-1,2,-2,3,-3,4,-4,5,-5\} \\
& f_{e}\left(E\left(S\left(P_{5} K_{1}\right)\right)\right)=f_{e}\left(E\left(S\left(P_{3} K_{1}\right)\right)\right) \\
& \cup\{18,21,23,25,-18,-21,-23,-25\}
\end{aligned}
$$

When $n>5$,

$$
\begin{aligned}
& f_{e}\left(E\left(S\left(P_{n} K_{1}\right)\right)\right)=\{26,31,33,35\} \\
& \cup\{-26,-31,-33,-35\} \cup\{36,41,43,45\} \\
& \cup\{-36,-41,-43,-45\} \cup, \cdots, \\
& \cup\{5 n-9,5 n-4,5 n-2,5 n\} \\
& \cup\{-(5 n-9),-(5 n-4),-(5 n-2),-5 n\} .
\end{aligned}
$$

Then $f$ is pair sum labeling.

Illustration 5. A pair sum labeling of $S\left(P_{8} K_{1}\right)$ is shown in Figure 5.

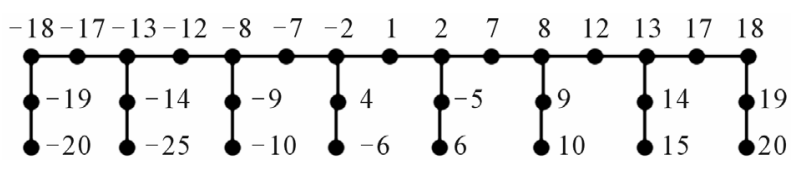

Figure 5. A pair sum labeling of $S\left(P_{8} K_{1}\right)$.

Theorem 4.4. $S\left(T_{n}\right)$ is a pair sum graph where $T_{n}$ is a triangular snake with $n$ triangle.

Proof. Let

$$
\begin{aligned}
& V\left(S\left(T_{n}\right)\right)=\left\{u_{i}: 1 \leq i \leq 2 n+1\right\} \\
& \cup\left\{v_{i}: 1 \leq i \leq 2 n\right\} \subset\left\{w_{i}: 1 \leq i \leq n\right\}
\end{aligned}
$$

and

$$
\begin{aligned}
& E\left(S\left(T_{n}\right)\right)=\left\{u_{i} u_{i+1}: 1 \leq i \leq 2 n\right\} \\
& \cup\left\{v_{2 i-1} w_{i}, v_{2 i} w_{i}: 1 \leq i \leq n\right\} \\
& \cup\left\{u_{2 i-1} v_{2 i-1}, u_{2 i+1} v_{2 i}: 1 \leq i \leq n\right\} .
\end{aligned}
$$

Case 1. $n$ is even.

When $n=2$, Define $f\left(u_{1}\right)=7, f\left(u_{2}\right)=6, f\left(u_{3}\right)=1, f\left(u_{4}\right)$ $=-6, f\left(u_{5}\right)=-7, f\left(v_{1}\right)=5, f\left(v_{2}\right)=2, f\left(v_{3}\right)=-4, f\left(v_{4}\right)=-5$, $f\left(w_{1}\right)=3, f\left(w_{2}\right)=-3$. When $n>2$, Define

$$
f: V\left(S\left(T_{n}\right)\right) \rightarrow\{ \pm 1, \pm 2, \cdots, \pm(5 n+1)\}
$$

by

$$
\begin{aligned}
& f\left(u_{n+1}\right)=1, f\left(u_{n}\right)=6 \\
& f\left(u_{n-1}\right)=7, f\left(u_{n+2}\right)=6 \\
& f\left(u_{n+3}\right)=-7 \\
& f\left(u_{n-2 i}\right)=10 i+6,1 \leq i \leq(n-2) / 2 \\
& f\left(u_{n-1-2 i}\right)=10 i+4,1 \leq i \leq(n-2) / 2 \\
& f\left(u_{n+2+2 i}\right)=-10 i-6,1 \leq i \leq(n-2) / 2 \\
& f\left(u_{n+3+2 i}\right)=-10 i-4,1 \leq i \leq(n-2) / 2 \\
& f\left(v_{n}\right)=2, f\left(v_{n-1}\right)=5 \\
& f\left(v_{n+1}\right)=-4, f\left(v_{n+2}\right)=-5 \\
& f\left(v_{n-2 i}\right)=10 i-2,1 \leq i \leq(n-2) / 2 \\
& f\left(v_{n-1-2 i}\right)=10 i+2,1 \leq i \leq(n-2) / 2 \\
& f\left(v_{n+1+2 i}\right)=-10 i+2,1 \leq i \leq(n-2) / 2 \\
& f\left(v_{n+2+2 i}\right)=-10 i-2,1 \leq i \leq(n-2) / 2 \\
& f\left(w_{n / 2}\right)=3, f\left(w_{(n+2) / 2}\right)=-3 \\
& f\left(w_{(n-2 i) / 2}\right)=10 i, 1 \leq i \leq(n-2) / 2 \\
& f\left(w_{n / 2+i+1}\right)=-10 i, 1 \leq i \leq(n-2) / 2 \\
& =0
\end{aligned}
$$

For $n=4$, 
$f_{e}\left(E\left(S\left(T_{n}\right)\right)\right)=\{3,5,7,8,12,13,15,18,22,23,26,30\}$

$\cup\{-3,-5,-7,-8,-12,-13,-15,-18,-22,-23,-26,-30\}$.

For $n>4$

$f_{e}\left(E\left(S\left(T_{n}\right)\right)\right)=f_{e}\left(E\left(S\left(T_{4}\right)\right)\right)$

$\cup\{(32,38,40,42,46,50),(-32,-38,-40,-42,-46,-50)$,

$(52,58,60,62,66,70),(-52,-58,-60,-62,-66,-70), \cdots$,

$(10 n-28,10 n-22,10 n-20,10 n-18,10 n-14,10 n-10)$,

$(-10 n+28,-10 n+22,-10 n+20$,

$-10 n+18,-10 n+14,-10 n+10)\}$.

Then $f$ is pair sum labeling.

Case 2. $n$ is odd.

Clearly $S\left(T_{1}\right) \cong C_{6}$, and hence $S\left(T_{1}\right)$ is a pair sum graph by Theorem 2.3.

For $>1$, Define

$$
f: V\left(S\left(T_{n}\right)\right) \rightarrow\{ \pm 1, \pm 2, \cdots, \pm(5 n+1)\} \cong C_{6}
$$

by

$$
\begin{aligned}
& f\left(u_{n+1}\right)=-8, f\left(u_{n}\right)=3 \\
& f\left(u_{n-1}\right)=-2, f\left(u_{n-2}\right)=-15 \\
& f\left(u_{n+2}\right)=-5, f\left(u_{n+3}\right)=4 \\
& f\left(u_{n+4}\right)=15 \\
& f\left(u_{n-1-2 i}\right)=-14-5 i, 1 \leq i \leq(n-3) / 2 \\
& f\left(u_{n-2-2 i}\right)=-10 i-4,1 \leq i \leq(n-3) / 2 \\
& f\left(u_{n+3+2 i}\right)=14+5 i, 1 \leq i \leq(n-3) / 2 \\
& f\left(u_{n+4+2 i}\right)=15+5 i, 1 \leq i \leq(n-3) / 2 \\
& f\left(v_{n}\right)=5, f\left(v_{n-1}\right)=-7 \\
& f\left(v_{n-2}\right)=-4, f\left(v_{n+1}\right)=-3 \\
& f\left(v_{n+2}\right)=7, f\left(v_{n+3}\right)=2 \\
& f\left(v_{n-1-2 i}\right)=-11-5 i, 1 \leq i \leq(n-3) / 2 \\
& f\left(v_{n-2-2 i}\right)=-5 i-13,1 \leq i \leq(n-3) / 2 \\
& f\left(v_{n+1+2 i}\right)=11+5 i, 1 \leq i \leq(n-3) / 2 \\
& f\left(v_{n+2+2 i}\right)=13+5 i, 1 \leq i \leq(n-3) / 2 \\
& f\left(w_{(n+1) / 2}\right)=8, f\left(w_{(n-1) / 2}\right)=11 \\
& f\left(w_{(n+3) / 2}\right)=-9, \\
& f\left(w_{(n-1) / 2-i}\right)=-12-5 i, 1 \leq i \leq(n-3) / 2
\end{aligned}
$$

$$
f\left(w_{(n+3) / 2+i}\right)=12+5 i, 1 \leq i \leq(n-3) / 2
$$

Here $n=3$,

$$
\begin{aligned}
& f_{e}\left(E\left(S\left(T_{n}\right)\right)\right)=\{1,2,4,5,7,8,13,17,19\} \\
& \cup\{-1,-2,-4,-5,-7-8,-13,-17,-19\} .
\end{aligned}
$$

For $n>3$,

$f_{e}\left(E\left(S\left(T_{n}\right)\right)\right)=f_{e}\left(E\left(S\left(T_{3}\right)\right)\right)$

$\cup\{(31,33,34,35,38,39),(-31,-33,-34,-35,-38,-39)$,

$(41,43,44,45,48,49),(-41,-43,-44,-45,-48,-49), \cdots$,

$(5 n+6,5 n+8,5 n+9,5 n+10,5 n+13,5 n+14)$,

$(-5 n-6,-5 n-8,-5 n-9,-5 n-10,-5 n-13,-5 n-14)\}$.

Then $f$ is pair sum labeling.

Illustration 6. A pair sum labeling of $S\left(T_{5}\right)$ is shown in Figure 6.

Theorem 4.5. $S\left(Q_{n}\right)$ is a pair sum graph.

Proof. Let

$$
\begin{aligned}
& V\left(S\left(Q_{n}\right)\right)=\left\{u_{i}: 1 \leq i \leq 2 n+1\right\} \\
& \cup\left\{v_{i}: 1 \leq i \leq 3 n\right\} \cup\left\{w_{i}: 1 \leq i \leq 2 n\right\}
\end{aligned}
$$

and

$$
\begin{aligned}
& E\left(S\left(Q_{n}\right)\right)\left\{u_{i} u_{i+1}: 1 \leq i \leq 2 n\right\} \\
& \cup\left\{u_{2 i+1} w_{2 i}, v_{3 i} w_{2 i}: 1 \leq i \leq n\right\} \\
& \cup\left\{u_{2 i-1} w_{2 i-1}, w_{2 i-1} v_{3 i-2}: 1 \leq i \leq n\right\} \\
& \cup\left\{v_{i} v_{i+1}: 1 \leq i \leq 3 n-1\right\}-\left\{v_{3 i} v_{3 i+1}: 1 \leq i \leq n\right\} .
\end{aligned}
$$

Case 1. $n$ is even.

When $n=2$, Define $f\left(u_{1}\right)=11, f\left(u_{2}\right)=6, f\left(u_{3}\right)=1$, $f\left(u_{4}\right)=-6, f\left(u_{5}\right)=-11, f\left(w_{1}\right)=9, f\left(w_{2}\right)=2, f\left(w_{3}\right)=-4$, $f\left(w_{4}\right)=-9, f\left(v_{1}\right)=7, f\left(v_{2}\right)=5, f\left(v_{3}\right)=3, f\left(v_{4}\right)=-3, f\left(v_{5}\right)=$ $-5, f\left(v_{6}\right)=-7$. When $>2$, Define

$$
f: V\left(S\left(Q_{n}\right)\right) \rightarrow\{ \pm 1, \pm 2, \cdots, \pm(7 n+1)\}
$$

by

$$
\begin{aligned}
& f\left(u_{n+1}\right)=1, f\left(u_{n-1}\right)=6 \\
& f\left(u_{n-2}\right)=8, f\left(u_{n+1}\right)=-6, f\left(u_{n+2}\right)=-8 \\
& f\left(u_{n-2-2 i}\right)=14 i+8,1 \leq i \leq(n-2) / 2 \\
& f\left(u_{n-1-2 i}\right)=14 i+6,1 \leq i \leq(n-2) / 2
\end{aligned}
$$

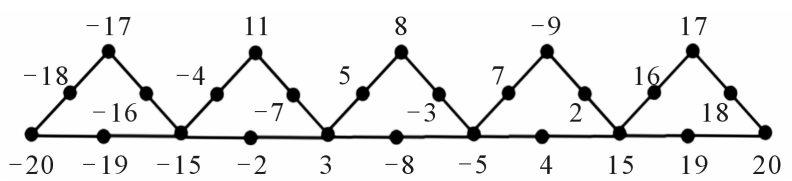

Figure 6. A pair sum labeling of $S\left(T_{5}\right)$. 


$$
\begin{aligned}
& f\left(u_{n+1+2 i}\right)=-14 i-6,1 \leq i \leq(n-2) / 2 \\
& f\left(u_{n+2+2 i}\right)=-14 i-8,1 \leq i \leq(n-2) / 2 \\
& f\left(w_{n}\right)=2, f\left(w_{n-1}\right)=9 \\
& f\left(w_{n+1}\right)=-4, f\left(w_{n+2}\right)=-9 \\
& f\left(w_{n-2 i}\right)=14 i-4,1 \leq i \leq(n-2) / 2 \\
& f\left(w_{n-1-2 i}\right)=14 i+4,1 \leq i \leq(n-2) / 2 \\
& f\left(w_{n+1+2 i}\right)=-14 i+4,1 \leq i \leq(n-2) / 2 \\
& f\left(w_{n+2+2 i}\right)=-14 i-4,1 \leq i \leq(n-2) / 2 \\
& f\left(v_{3 n / 2}\right)=3, f\left(v_{3 n-1 / 2}\right)=5 \\
& f\left(v_{3 n-2 / 2}\right)=7, f\left(v_{3 n+1 / 2}\right)=-3 \\
& f\left(v_{3 n+2 / 2}\right)=-5, f\left(v_{3 n+3 / 2}\right)=-7 \\
& f\left(v_{3 n / 2-3 i}\right)=14 i-2,1 \leq i \leq(n-2) / 2 \\
& f\left(v_{3 n / 2-2-3 i}\right)=14 i, 1 \leq i \leq(n-2) / 2 \\
& f\left(v_{3 n / 2-1-3 i}\right)=14 i+2,1 \leq i \leq(n-2) / 2 \\
& f\left(v_{3 n / 2+1+3 i}\right)=-14 i+2,1 \leq i \leq(n-2) / 2 \\
& f\left(v_{3 n / 2+2+3 i}\right)=-14 i, 1 \leq i \leq(n-2) / 2 \\
& f\left(v_{3 n / 2+3+3 i}\right)=-14 i-2,1 \leq i \leq(n-2) / 2
\end{aligned}
$$

Here

$$
\begin{aligned}
& f_{e}\left(E\left(S\left(Q_{n}\right)\right)\right)=\{3,-3,5,-5,7,-7,8,-8, \\
&12,-12,14,-1416,-16,17,-17\} \\
& \cup\{18,22,26,28,30,34,40,42\} \\
& \cup\{-18,-22,-26,-28,-30,-34,-40,-42\} \\
& \cup\{46,50,54,56,58,62,68,70\} \\
& \cup\{-46,-50,-54,-56,-58,-62,-68,-70\} \\
& \cup\{74,78,82,86,84,90,96,98\} \\
& \cup\{-74,-78,-82,-86,-84,-90,-96,-98\} \bigcup \cdots, \\
& \cup\{14 n-38,14 n-34,14 n-30,14 n-28, \\
&14 n-26,14 n-22,14 n-16,14 n-14\} \\
& \cup\{-14 n+38,-14 n+34,-14 n+30,-14 n+28, \\
&-14 n+26,-14 n+22,-14 n+16,-14 n+14\} .
\end{aligned}
$$

Then $f$ is pair sum labeling.

Case 2. $n$ is odd.

$S\left(Q_{1}\right)$ is a pair sum graph follows from Theorem 2.3. When $n>1$. Define

$$
f: V\left(S\left(Q_{n}\right)\right) \rightarrow\{ \pm 1, \pm 2, \cdots, \pm(7 n+1)\}
$$

by

$$
\begin{aligned}
& f\left(u_{n+1}\right)=-6, f\left(u_{n}\right)=7, f\left(u_{n-1}\right)=8 \\
& f\left(u_{n-2}\right)=22, f\left(u_{n+2}\right)=-3, f\left(u_{n+3}\right)=-8 \\
& f\left(u_{n+4}\right)=-22 \\
& f\left(u_{n-1-2 i}\right)=14 i+20,1 \leq i \leq(n-3) / 2 \\
& f\left(u_{n-2-2 i}\right)=14 i+22,1 \leq i \leq(n-3) / 2 \\
& f\left(u_{n+3+2 i}\right)=-14 i-20,1 \leq i \leq(n-3) / 2 \\
& f\left(u_{n+4+2 i}\right)=-14 i-22,1 \leq i \leq(n-3) / 2 \\
& f\left(w_{n}\right)=5, f\left(w_{n-1}\right)=4, f\left(w_{n-2}\right)=20 \\
& f\left(w_{n+1}\right)=-5, f\left(w_{n+2}\right)=-12, f\left(w_{n+3}\right)=-20 \\
& f\left(w_{n-1-2 i}\right)=14 i+10,1 \leq i \leq(n-3) / 2 \\
& f\left(w_{n-2-2 i}\right)=18+14 i, 1 \leq i \leq(n-3) / 2 \\
& f\left(w_{n+2+2 i}\right)=-14 i-10,1 \leq i \leq(n-3) / 2 \\
& f\left(w_{n+3+2 i}\right)=-18-14 i, 1 \leq i \leq(n-3) / 2 \\
& f\left(v_{(3 n+1) / 2}\right)=6, f\left(v_{(3 n-1) / 2}\right)=3 \\
& f\left(v_{(3 n-3) / 2}\right)=-10, f\left(v_{(3 n-5) / 2}\right)=16 \\
& f\left(v_{(3 n-7) / 2}\right)=18, f\left(v_{(3 n+3) / 2}\right)=-7 \\
& f\left(v_{(3 n+5) / 2}\right)=14, f\left(v_{(3 n+7) / 2}\right)=-16 \\
& f\left(v_{(3 n+9) / 2}\right)=-18 \\
& f\left(v_{(3 n-3) / 2-3 i}\right)=14 i+12,1 \leq i \leq(n-3) / 2 \\
& f\left(v_{(3 n-5) / 2-3 i}\right)=14 i+14,1 \leq i \leq(n-3) / 2 \\
& f\left(v_{(3 n-7) / 2-3 i}\right)=14 i+6,1 \leq i \leq(n-3) / 2 \\
& f\left(v_{(3 n+5) / 2+3 i}\right)=-14 i-12,1 \leq i \leq(n-3) / 2 \\
& f\left(v_{(3 n+7) / 2+3 i}\right)=-14 i-14,1 \leq i \leq(n-3) / 2 \\
& f\left(v_{(3 n+9) / 2+3 i}\right)=-14 i-6,1 \leq i \leq(n-3) / 2 \\
&
\end{aligned}
$$

For $n=3$,

$f_{e}\left(E\left(S\left(Q_{n}\right)\right)\right)=\{1,2,6,8,9,11,12,15,30,34,38,42\}$

$\cup\{-1,-2,-6,-8,-9,-11,-12,-15,-30,-34,-38,-42\}$.

$n>3$, 


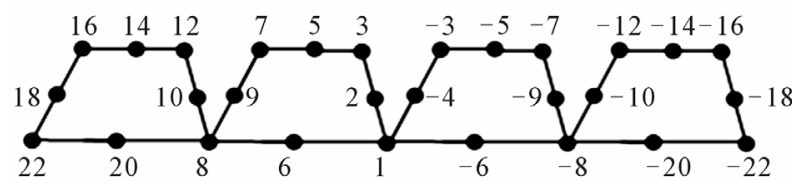

Figure 7. A pair sum labeling of $S\left(Q_{4}\right)$.

$$
\begin{aligned}
& f_{e}\left(E\left(S\left(Q_{n}\right)\right)=f_{e}\left(E\left(S\left(Q_{3}\right)\right)\right)\right. \\
& \cup\{(46,50,54,56,58,62,68,70), \\
& (-46,-50,-54,-56,-58,-62,-68,-70), \\
& (74,78,82,84,86,90,96,98), \\
& (-74,-78,-82,-84,-86,-90,-96,-98), \cdots, \\
& (14 n-24,14 n-20,14 n-16,14 n-14, \\
& 14 n-12,14 n-8,14 n-2,14 n) \\
& (-14 n+24,-14 n+20,-14 n+16,-14 n+14, \\
& -14 n+12,-14 n+8,-14 n+2,-14 n)\} .
\end{aligned}
$$

Then $f$ is pair sum labeling

Illustration 7. A pair sum labeling of $S\left(Q_{4}\right)$ is shown in Figure 7.

\section{Acknwledgements}

We thank the referees for their valuable comments and suggestions.

\section{REFERENCES}

[1] R. Ponraj and J. V. X. Parthipan, "Pair Sum Labeling of Graphs," The Journal of Indian Academy of Mathematics, Vol. 32, No. 2, 2010, pp. 587-595.

[2] R. Ponraj, J. V. X. Parthipan and R. Kala, "Some Results on Pair Sum Labeling," International Journal of Mathematical Combinatorics, Vol. 4, 2010, pp. 53-61.

[3] R. Ponraj, J. V. X. Parthipan and R. Kala, "A Note on Pair Sum Graphs," Journal of Scientific Research, Vol. 3, No. 2, 2011, pp. 321-329.

[4] R. Ponraj and J. V. X. Parthipan, "Further Results on Pair Sum Labeling of Trees," Applied Mathematics, Vol. 2, No. 10, 2011, pp. 1270-1278. doi:10.4236/am.2011.210177

[5] F. Harary, "Graph Theory," Narosa Publishing House, New Delhi, 1998. 